\title{
Perception of Emotion in Torso and Arm Movements on Humanoid Robot Quori
}

\author{
Roshni Kaushik \\ roshnika@andrew.cmu.edu \\ Carnegie Mellon University \\ Pittsburgh, PA, USA
}

\author{
Reid Simmons \\ rsimmons@andrew.cmu.edu \\ Carnegie Mellon University \\ Pittsburgh, PA, USA
}

\begin{abstract}
Displaying emotional states is an important part of nonverbal communication that can facilitate successful interactions. Facial expressions have been studied for their emotional expression, but this work looks at the capacity of body movements to convey different emotions. This work first generates a large set of nonverbal behaviors with a variety of torso and arm properties on a humanoid robot, Quori. Participants in a user study evaluated how much each movement displayed each of eight different emotions. Results indicate that specific movement properties are associated with particular emotions; such as leaning backward and arms held high displaying surprise and leaning forward displaying sadness. Understanding the emotions associated with certain movements can allow for the design of more appropriate behaviors during interactions with humans and could improve people's perception of the robot.
\end{abstract}

\section{CCS CONCEPTS}

- Human-centered computing $\rightarrow$ Collaborative and social computing design and evaluation methods; HCI design and evaluation methods.

\section{KEYWORDS}

nonverbal communication; expressive motion; emotion recognition

\section{ACM Reference Format:}

Roshni Kaushik and Reid Simmons. 2021. Perception of Emotion in Torso and Arm Movements on Humanoid Robot Quori. In Companion of the 2021 ACM/IEEE International Conference on Human-Robot Interaction (HRI '21 Companion), March 8-11, 2021, Boulder, CO, USA. ACM, New York, NY, USA, 5 pages. https://doi.org/10.1145/3434074.3447129

\section{INTRODUCTION}

Nonverbal communication is an important aspect of successful teaching. It can serve a variety of purposes including supplementing verbal exchanges, revealing emotional states, and influencing the performance of others [25]. Student nonverbal behaviors such as attentiveness and use of space can also help teachers be more successful in teaching [13].

Our work is motivated by the development of a robotic student. Peer tutoring can be an effective strategy for solidifying learning

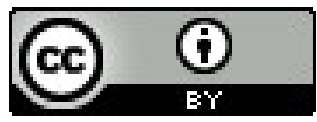

This work is licensed under a Creative Commons Attribution International 4.0 License.

HRI '21, March 8-11, 2021, Boulder, CO, USA.

(C) 2021 Copyright held by the owner/author(s).

ACM ISBN 978-1-4503-8289-2/21/03. https://doi.org/10.1145/3434074.3447129 gains [17]. There has been much study into how robots can tutor children [19], but we are interested in the reverse direction; how a student can tutor a robot. To move towards this goal, the robot being tutored must be able to respond emotionally to the student as human students do to their teachers. The successful identification of a robot's nonverbally displayed emotions creates the potential for an elicited a human emotional response and for the human to feel more connected with the robot and engaged in their task [18].

Our goal in this work, therefore, is to develop nonverbal robot movements for the Quori robot (Figure 1) to display specific emotions. This robot has a waist joint allowing it to lean forward and backward, movement that has been shown to correlate with various emotional behaviors [2-4]. Although emotional behaviors are composed of many modalities (speech, movement, facial expressions, etc.), we focus on body movements exclusively in this work to explore how even low-DOF motion can convey emotions.

After reviewing existing literature on how humans and robots use nonverbal movements to display emotion, we chose a computational approach inspired by [21] and generated 224 movements by combining torso and arm degrees-of-freedom (DOF) of the Quori robot. We administered a survey to 145 online participants to evaluate to what extent each of these movements displayed 8 emotional states. We compared these perceptions of robot emotion to how humans display those same emotions through their nonverbal behavior, and our results indicate many similarities in how emotions are expressed in humans and Quori.

\section{NONVERBAL MOVEMENTS EXPRESSING EMOTIONS}

We will discuss how humans and robots use nonverbal movements to display various emotions, focusing on educational applications whenever possible. Nonverbal communication is defined as the process of stimulating meaning in the minds of others through messages that are nonlinguistic or non-language based [16]. This form of communication is composed of several categories including kinesics (body motions, posture, facial expressions, etc.), paralanguage (voice volume, pitch, tempo, etc.), and proxemics (use of space) [22]. In students, nonverbal messages such as a slouching position can indicate a lack of interest and leaning forward can show attentiveness [13]. Nonverbal communication can also convey emotions, with some research suggesting that up to $90 \%$ of emotional meaning is conveyed through nonverbal behaviors [12].

Robots expressing emotions is a highly studied area for both humanoid and non-humanoid robots. Researchers investigated how changing the poses of the NAO humanoid robot affected the perception of the robot's affect [1]. This work looks at creating an Affect Space where the nonverbal behaviors of the robot map to 
Table 1: Torso and arm movements from literature associated with 7 emotions

\begin{tabular}{ll}
\hline Emotion & From Literature \\
\hline \multirow{2}{*}{ Happiness } & $\begin{array}{l}\text { Symmetrical up-down motion of arms [4]; Hands kept high, hands made into fists and kept high [24]; } \\
\text { Slight lean backwards, arms raised high [3] }\end{array}$ \\
\hline Sadness & Leaning forward, Hands at sides [2]; Hands over head [24]; Leaning forward, Hands at sides [3] \\
\hline Fear & $\begin{array}{l}\text { Leaning backward [4]; Hands out to sides [2]; Body backing, Hands over head, trying to cover body [24]; } \\
\text { Leaning backward, Arms slightly forward [3] }\end{array}$ \\
\hline Disgust & Leaning backward, Arms forward [3] \\
\hline Anger & $\begin{array}{l}\text { Leaning forward [4]; Leaning forward, Arms crossed, on hips [2]; Hands on waist, hands into fist or low, } \\
\text { fast hand lift [24]; Leaning forward, Arms forward [3] }\end{array}$ \\
\hline Surprise & Hands over head [24]; Leaning backward, Hands over head [3] \\
\hline Interest & Leaning forward, Arms resting at side [4] \\
\hline
\end{tabular}

perceived emotion, with a mapping different from that for humans. Humans rely on a combination of many features to display emotion including facial expressions [5], which have been decomposed into Facial Action Units in [6] to display various emotions.

However, a robot has a different morphology and capabilities to a human, so must use its unique composition to display emotion. In our work, we attempt a similar process to the Affect Space, but with dynamic movements of the Quori robot, and focusing on torso and arm movement exclusively, as these two types of movements are the only DOF available aside from translation and rotation of the entire robot.

Robots performing a teaching role have used nonverbal behavior to improve the students' experience. Participants found that a robot programmed to have a positive mood increased the valence and arousal of its audience compared to using a negative mood [27]. Researchers have also developed a model for a NAO robot teacher to express different levels of warmth and competence with its body postures and hand gestures [15].

Emotional nonverbal behavior has also been studied outside of a specific educational context. Using designed body language for a humanoid robot, researchers tested emotion recognition [11]. Emotional behavior by a humanoid robot (NAO or Mini Darwin Platform) was developed to assist autistic children with emotion recognition [7]. Researchers developed a framework for displaying robot traits, moods, and emotions using nonverbal behaviors [14].

Laban movement analysis [10] is an approach to movement parameterization extensively used, including to convey affect through flight paths [20]. Using Laban efforts, head movements were used to convey a robot state using Keepon and NAO robots [9], and the path of mobile robots was used to create expressive motion [8]. In our work, we parameterize torso and arm movements with various properties to determine which properties correlate with each emotion. Similarly, researchers parameterized hand movements (e.g. waving) by pose and motion parameters that participants used to create movements corresponding to different moods [26].

We can only modify torso and arm DOF of our robot, so we focus our attention on which kinds of torso and arm movements on humans from literature are correlated with displaying different emotions, again focusing on educational domains whenever possible. Table 1 summarizes our review of this literature with specific movement descriptions for each of seven emotions. These are the six basic expressions of happiness, sadness, fear, disgust, anger, and surprise from [6], with the addition of interest which is useful in educational contexts.

\section{GENERATING ROBOT MOVEMENTS}

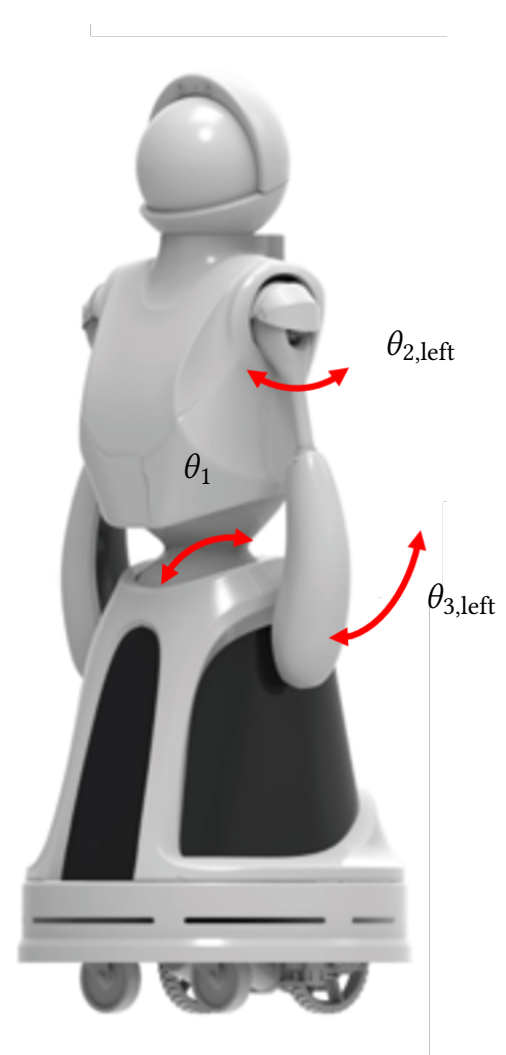

Figure 1: Torso and left arm angles labelled on the Quori robot (image from http://www.quori.org/)

Quori (Figure 1) is a robot designed for and by the HRI community [23]. It has many features including a waist that can bend (a rarity in most currently available robots) and gesturing arms. Due to COVID, the robot is not yet available to us, so we developed a simulation environment using ROS/Gazebo that allowed us to command the arms and torso to a desired position at a specified 
speed (maximum of $1 \mathrm{rad} / \mathrm{s}$ for all joints). The torso has one DOF $\left(\theta_{1}\right)$ and can lean forward to $0.47 \mathrm{rad}$ and backward to $-0.21 \mathrm{rad}$. Each arm has two co-located joints: a rotational shoulder joint with no joint limits $\left(\theta_{2, \text { left/right }}\right)$ and a lifting shoulder joint with $\pm 1.1 \mathrm{rad}$ limits $\left(\theta_{3, \text { left } / \text { right }}\right)$.

To constrain the large number of possible movements created by combining these DOF, we developed a set of design considerations. For torso motion, we only created movements starting at a neutral position $\left(\theta_{1}=0\right)$ and ending forward or backward at a small or large angle. The torso could move between its start and end position at a slow or a fast speed. The discretization of the possible end positions to 4 possibilities and the speeds to 2 levels allowed us to reduce the number of possibilities but still include a variety of torso movements.

When choosing possible arm movements, we first determined whether the movement would be symmetric or asymmetric. For symmetric movements, both arms would start forward or at the robot's sides and could end either forward, at the robot's sides, or above the robot's head. This motion could be performed at a slow or a fast speed. For asymmetric movements, the left arm was stationary either forward, at the robot's side, or above its head. The right arm could be stationary in either the forward or side position. It could also move from a forward or side position to a forward, side, or high position, at a slow or fast speed. By only including asymmetric movements with the left arm stationary, we eliminated the mirror-image movement with the right arm stationary and left arm moving. We believe that the emotion perceived in the movement is agnostic to which arm is moving. We also chose not to include movements with both arms moving asymmetrically as this would exponentially increase the number of movements to evaluate.

After applying these constraints, we categorize the resulting 224 movements using the 6 properties shown in Table 2, with each movement taking one value for each property. We decided not to analyze differences due to arm start position, so that property is not listed in this table.

Table 2: Movement Properties

\begin{tabular}{l|lll}
\hline Property & Value 1 & Value 2 & Value 3 \\
\hline Torso End & forward & backward & \\
Torso Degree & small & large & \\
Torso Speed & slow & fast & \\
(Arm) Symmetry & true & false & \\
(Right) Arm End & forward & sides & high \\
(Right) Arm Speed & slow & fast & \\
\hline
\end{tabular}

\section{SURVEY AND DATA ANALYSIS}

We developed a survey to test if these programmatically-generated 224 movements were perceived as displaying emotion. This survey, created using Qualtrics and administered using Amazon Mechanical Turk, presented 10 randomly selected videos to 145 participants. Due to the randomization, each video was not seen the same number of times, but on average was seen 6-7 times. These participants were mostly in the 25-44 age range with a varied familiarity with robots. $60 \%$ identified as male and $80 \%$ as white.
For each video, the participants were asked, "How much is the robot expressing each emotion?" for the 7 emotions shown in Table 1 plus Neutral. For each of the 8 emotions, the participants chose from a 5-point Likert scale with labels Not, Slightly, Somewhat, Moderately, and Intensely to indicate their perceived intensity of that emotion in the robot movement. We chose to collect the participants' perceptions using a set of Likert scales to allow for multiple emotions to be seen at different intensities in the same robot movement. This also allows for no or very little emotion to be seen in a movement, as opposed to other question styles that may force the participant to chose a single emotion seen in a movement.

Before this set of 10 questions, each participant confirmed consent to participate in the study and saw a training video (not out of the 224 videos) to practice answering the Likert-style questions. At the end of the survey, we asked a series of demographic questions, including age, gender, ethnicity, and familiarity with robots (chosen on a 5-point Likert scale).

To determine whether a movement on Quori will be perceived as conveying the same emotion as a similar human movement (Table 1), we need to determine the movement properties that particular emotion was associated with from our survey results. We first grouped the movements by value for a specific property. For the first property, we have a group of movements with the torso forward and a second group with the torso backward. We want to see if emotions were perceived significantly differently between these two groups. To allow for the same participant to see multiple movements from the same group, we performed a Friedman Test with a Neymeni post-hoc that includes a blocking variable (the participant). For each emotion, we compared the Likert values (as ordinal, categorical variables) between groups. The output of this statistical test will be a p-value, with a value below 0.05 indicating the two groups are significantly different. We repeated these comparisons, comparing the distributions of Likert values between the different options, for each property.

\section{RESULTS}

As described in the Data Analysis section, we compared the distribution of Likert responses between values in each property (i.e. torso forward vs. torso backward, arm slow vs. arm fast). The results of this analysis is shown in Table 1 . This table combines two kinds of information: (1) the significant survey results with p-values below 0.05 and (2) the literature review information (Table 1).

We can see that certain emotions had many more significant results from the survey than others (black, unitalicized text). Surprise and Sadness each had many properties with significant results; Happiness and Fear had few properties; and Disgust, Anger, Interest, and Neutral had either 1 or no discriminating properties. This may point to the need for additional DOF or variety in the robot movements to display these emotions perceptibly. It may also indicate that the emotions that had few significant properties are in general more difficult to recognize due to variety in their expression in humans due to cultural or contextual differences.

We can also see that certain movement properties were more distinctive to display emotion. Specifically, the end of the arm movements and the end of the torso movement have significant values for many emotions, as compared to torso degree and torso speed. 
Table 3: The value in each cell indicates the value of the property (Table 2) that was either described in the literature (Table 1) to be associated with a particular emotion (blue, italicized text) and/or had the highest Likert score compared to movements with a different value of that property (black, unitalicized text).

\begin{tabular}{|c|c|c|c|c|c|c|}
\hline & Torso End & Torso Degree & Torso Speed & (Arm) Symmetry & (Right) Arm End & (Right) Arm Speed \\
\hline Happiness & Backward & & & $\begin{array}{c}\text { Symmetric }(\mathrm{p}=0.037) \\
\text { Symmetric }\end{array}$ & $\begin{array}{c}\text { High }(\mathrm{p}=0.001) \\
\text { High }\end{array}$ & \\
\hline Sadness & $\begin{array}{l}\text { Forward }(\mathrm{p}=0.001) \\
\text { Forward }\end{array}$ & Large $(p=0.001)$ & & & $\begin{array}{c}\text { Forward, Sides } \\
(\mathrm{p}=0.001) \\
\text { Forward, Sides, High }\end{array}$ & Slow $(p=0.004)$ \\
\hline Fear & Backward & & Fast $(\mathrm{p}=0.073)$ & & $\begin{array}{c}\text { Forward }(\mathrm{p}=0.001) \\
\text { Forward, Sides, High }\end{array}$ & \\
\hline Disgust & Backward & & Fast $(\mathrm{p}=0.007)$ & & Forward & \\
\hline Anger & Forward & & & & Forward & Fast \\
\hline Surprise & $\begin{array}{c}\text { Backward }(\mathrm{p}=0.001) \\
\text { Backward }\end{array}$ & Large $(\mathrm{p}=0.01)$ & & Symmetric $(p=0.006)$ & $\begin{array}{c}\text { High }(\mathrm{p}=0.001) \\
\text { High }\end{array}$ & Fast $(p=0.005)$ \\
\hline Interest & & & & & $\begin{array}{c}\text { High, Forward } \\
(\mathrm{p}=0.002) \\
\text { Sides }\end{array}$ & \\
\hline \multicolumn{7}{|l|}{ Neutral } \\
\hline Key: & $\begin{array}{l}\text { Literature and } \\
\text { Survey agree }\end{array}$ & $\begin{array}{l}\text { Literature and } \\
\text { Survey disagree }\end{array}$ & Survey & Literature & & \\
\hline
\end{tabular}

This could indicate specific types of movement convey emotions more clearly than others, although interactions between movement properties can complicate this trend.

We are interested to see where the survey and literature results agree and disagree. As seen by the large number of green colored squares in the table, there are many cases where these two agree, especially in the end position of the arms and the torso end position. Only in one case did the two disagree (red background, dark left border), which is the end position of the arms for displaying Interest. This disagreement could be due to there existing a large variety of behaviors that are associated with human's display of Interest. For example, raising one's hand (arm position high) and reaching out to shake someone's hand (arm position forward) could be considered displaying Interest in specific situations. A difference in contexts between the emotional expression from the literature and Quori's movements could also be a source of this discrepancy.

We additionally looked at demographic trends in the results. We found that those familiar with robots and those identifying as Black or African American saw significantly more emotion in the robot movements. Participants identifying as male saw more of the negative valence emotions (Sadness, Fear, and Disgust) compared to those identifying as female. These results indicate that there are significant differences in how different demographic groups view robot movements, and factors such as robot familiarity can have a considerable effect (perhaps more than even the direction of the torso) on how these movements are perceived.

\section{CONCLUSION}

This paper presents a user-driven approach for evaluating the perceived emotion in a large set of nonverbal behaviors on the humanoid robot Quori. We compared the results of our survey with human torso and arm movement in literature shown to be associated with emotions and found many parallels. We found that certain movement properties, such as the torso leaning backward or the arms ending high, were associated with particular emotions.

This work has several limitations. First, the simulated robot used here may have a different effect compared to the physically present robot. The data analysis presented does assume that when comparing groups of movements across different possible values of a movement property, the remaining properties are sufficiently varied within the group to allow for that comparison. Despite these limitations, our work shows strong correlations between what is found in literature for emotion and how people perceive emotion on our humanoid robot.

Future extensions of this work are two-fold. First, because some emotions, such Anger and Interest, had very few distinguishing properties, we can conclude that it takes something more to characterize these emotions on Quori. Adding additional DOF can help humans distinguish these emotions correctly. Second, the robot was moving in isolation in this study with no context. Placing the robot in a human-robot interaction scenario in which it reacts appropriately to the human with these emotional nonverbal behaviors can allow us to test the effectiveness of the robot's emotional display more accurately.

\section{ACKNOWLEDGMENTS}

We thank Adrian Thinnyun for his development of the ROS/Gazebo simulation environment that allowed us to generate Quori's movements for this study. This work was supported in part by NSF Grant \#IIS-1939047, and the user study was approved under STUDY2020_00000048. 


\section{REFERENCES}

[1] Aryel Beck, Lola Cañamero, and Kim A. Bard. 2010. Towards an Affect Space for Robots to Display Emotional Body Language. In 19th International Symposium in Robot and Human Interactive Communication. IEEE, 464-469.

[2] Stéphanie Buisine, Matthieu Courgeon, Aurélien Charles, Céline Clavel, JeanClaude Martin, Ning Tan, and Ouriel Grynszpan. 2014. The Role of Body Postures in the Recognition of Emotions in Contextually Rich Scenarios. International Journal of Human-Computer Interaction 30, 1 (2014), 52-62.

[3] Mark Coulson. 2004. Attributing Emotion to Static Body Postures: Recognition Accuracy, Confusions, and Viewpoint Dependence. Fournal of nonverbal behavior 28,2 (2004), 117-139.

[4] Nele Dael, Marcello Mortillaro, and Klaus R. Scherer. 2012. Emotion Expression in Body Action and Posture. Emotion 12, 5 (2012), 1085.

[5] Paul Ekman. 1993. Facial Expression and Emotion. American psychologist 48, 4 (1993), 384.

[6] Paul Ekman and Wallace V. Friesen. 1978. Facial Action Coding Systems. Consulting Psychologists Press.

[7] Brittney A. English, Alexis Coates, and Ayanna Howard. 2017. Recognition of Gestural Behaviors Expressed by Humanoid Robotic Platforms for Teaching Affect Recognition to Children with Autism-a Healthy Subjects Pilot Study. In International Conference on Social Robotics. Springer, 567-576.

[8] Heather Knight and Reid Simmons. 2014. Expressive Motion with x, y and Theta: Laban Effort Features for Mobile Robots. In The 23rd IEEE International Symposium on Robot and Human Interactive Communication. IEEE, 267-273.

[9] Heather Knight and Reid Simmons. 2016. Laban Head-Motions Convey Robot State: A Call for Robot Body Language. In 2016 IEEE International Conference on Robotics and Automation (ICRA). IEEE, 2881-2888.

[10] Rudolf Laban and Lisa Ullmann. 1971. The Mastery of Movement. (1971).

[11] Derek McColl and Goldie Nejat. 2014. Recognizing Emotional Body Language Displayed by a Human-like Social Robot. International fournal of Social Robotics 6, 2 (2014), 261-280.

[12] Albert Mehrabian. 1971. Silent Messages. Vol. 8. Wadsworth Belmont, CA.

[13] TIMOTHY P. Mottet and VIRGINIA P. Richmond. 2002. Student Nonverbal Communication and Its Influence on Teachers and Teaching. Communication for teachers (2002), 47-61.

[14] Sunghyun Park, Lilia Moshkina, and Ronald C. Arkina. 2010. Recognizing Nonverbal Affective Behavior in Humanoid Robots. In Intelligent Autonomous Systems, Vol. 11. 12
[15] Rifca Peters, Joost Broekens, and Mark A. Neerincx. 2017. Robots Educate in Style: The Effect of Context and Non-Verbal Behaviour on Children's Perceptions of Warmth and Competence. In 2017 26th IEEE International Symposium on Robot and Human Interactive Communication (RO-MAN). IEEE, 449-455.

[16] Virginia P. Richmond, James C. McCroskey, and Mark Hickson. 2008. Nonverbal Behavior in Interpersonal Relations. Allyn \& Bacon.

17] Rod D. Roscoe and Michelene TH Chi. 2007. Understanding Tutor Learning: Knowledge-Building and Knowledge-Telling in Peer Tutors' Explanations and Questions. Review of educational research 77, 4 (2007), 534-574.

[18] Shane Saunderson and Goldie Nejat. 2019. How Robots Influence Humans: A Survey of Nonverbal Communication in Social Human-Robot Interaction. International fournal of Social Robotics 11, 4 (2019), 575-608.

[19] Sofia Serholt and Wolmet Barendregt. 2016. Robots Tutoring Children: Longitudinal Evaluation of Social Engagement in Child-Robot Interaction. In Proceedings of the 9th Nordic Conference on Human-Computer Interaction. ACM, 64.

[20] Megha Sharma, Dale Hildebrandt, Gem Newman, James E. Young, and Rasit Eskicioglu. 2013. Communicating Affect via Flight Path: Exploring Use of the Laban Effort System for Designing Affective Locomotion Paths. In Proceedings of the 8th ACM/IEEE International Conference on Human-Robot Interaction. IEEE Press, 293-300.

[21] Mohammad Shayganfar, Charles Rich, and Candace L. Sidner. 2012. A Design Methodology for Expressing Emotion on Robot Faces. In 2012 IEEE/RS7 International Conference on Intelligent Robots and Systems. IEEE, 4577-4583.

[22] Howard A. Smith. 1979. Nonverbal Communication in Teaching. Review of Educational Research 49, 4 (1979), 631-672.

[23] Andrew Specian, Nick Eckenstein, Mark Yim, Ross Mead, Braden McDorman, Simon Kim, and Maja Matarić. 2018. Preliminary System and Hardware Design for Quori, a Low-Cost, Modular, Socially Interactive Robot. In Workshop on Social Robots in the Wild.

[24] Ioanna-Ourania Stathopoulou and George A. Tsihrintzis. 2011. Emotion Recognition from Body Movements and Gestures. In Intelligent Interactive Multimedia Systems and Services. Springer, 295-303.

[25] Anita E. Woolfolk and Douglas M. Brooks. 1983. Chapter 5: Nonverbal Communication in Teaching. Review of research in education 10, 1 (1983), 103-149.

[26] Junchao Xu, Joost Broekens, Koen Hindriks, and Mark A. Neerincx. 2013. Mood Expression through Parameterized Functional Behavior of Robots. IEEE.

[27] Junchao Xu, Joost Broekens, Koen Hindriks, and Mark A. Neerincx. 2014. Effects of Bodily Mood Expression of a Robotic Teacher on Students. In 2014 IEEE/RSF International Conference on Intelligent Robots and Systems. IEEE, 2614-2620. 